\title{
An unusual case of mesenteric ischaemia
}

\author{
L R O Ayres, ${ }^{1,2}$ M Scott, ${ }^{3}$ N Shepherd, ${ }^{4}$ J Brown ${ }^{1}$
}

\begin{abstract}
${ }^{1}$ Department of
Gastroenterology, Gloucestershire Hospitals NHS Foundation Trust, Gloucester, UK

${ }^{2}$ Department of Gastroenterology, Fremantle Hospital, Western Australia, Australia

${ }^{3}$ Department of Surgery, Gloucestershire Hospitals NHS Foundation Trust, Gloucester, UK ${ }^{4}$ Department of Pathology, Gloucestershire Hospitals NHS Foundation Trust, Gloucester, UK

\section{Correspondence to:}

Dr Lachlan Richard Owen Ayres, Department of Gastroenterology, Fremantle Hospital, Alma Street Fremantle, Western Australia, WA 6160;

lachlanayres@hotmail.com
\end{abstract}

Received 30 July 2013 Revised 26 August 2013 Accepted 29 August 2013 Published Online First 18 September 2013
To cite: Ayres LRO, Scott M, Shepherd N, et al. Frontline Gastroenterology 2014;5: 36-39.

\section{ABSTRACT}

We describe an unusual cause of diarrhoea and segmental colitis in a previous well adult man.

Mesenteric inflammatory veno-occlusive disease is a rare cause of gastrointestinal tract ischaemia of unknown aetiology. We review the literature of this condition and other mesenteric venous pathologies.

\section{CASE HISTORY}

A 52-year-old man was admitted under the gastroenterology team with a 4 month history of watery diarrhoea, abdominal discomfort, bloating and weight loss. His past medical history included suspected glaucoma and bilateral hip replacements. He did not take any prescribed or over-the-counter medications.

Several weeks before admission he was investigated in the outpatient department by the surgical team. A barium enema, stool cultures and tissue transglutaminase antibody had been negative. Computed tomography (CT) of the abdomen showed diffuse thickening of the sigmoid colon and mild oedema throughout the mesosigmoid, with a maximal bowel wall thickness of $12 \mathrm{~mm}$. Colonoscopy showed slightly oedematous rectosigmoid mucosa. Biopsies from this area showed mild architectural distortion with a degree of crypt hyperplasia and mucin depletion. There was no inflammatory cell infiltrate and no evidence of idiopathic inflammatory bowel disease (IBD). Sequential empirical trials of metronidazole and 5-aminosalicylates to treat possible infection or IBD had no effect. Loperamide also had no effect.

Admission was prompted by worsening diarrhoea (every hour) and further weight loss. Examination revealed an afebrile, well-looking patient with a fullness in the right iliac fossa. Initial investigations showed haemoglobin $12.6 \mathrm{~g} / \mathrm{dL}$, WCC $9.3 \times 10^{9} / \mathrm{L}$, neutrophils
$7.74 \times 10^{9} / \mathrm{L}$, platelets 280 , CRP $11 \mathrm{mg} / \mathrm{L}$, albumin $34 \mathrm{~g} / \mathrm{L}$, TSH normal and stool culture negative. An autoimmune profile including antineutrophil cytoplasmic antibody was negative. Repeat CT abdomen and pelvis showed sigmoid colon thickening but no evidence of perforation or small bowel abnormality.

A flexible sigmoidoscopy showed an unusual appearance with marked oedema and a narrowed lumen from the sigmoid onwards. Enhanced vascular pattern and erythema in the rectum were noted (figure 1). The mucosa was firm on biopsy. Pending the histology result the patient was treated empirically with intravenous steroids and a further course of antibiotics (ciprofloxacin and metronidazole). The differential at this point included atypical IBD, infection or gastrointestinal vasculitis.

Histology showed changes of mucosal oedema associated with crypt architectural distortion and epithelial hyperplasia. Proliferation of the lamina propria including small blood vessels was also noted raising the possibility of subacute ischaemic colitis. However assessment with CT angiography failed to show evidence of arterial disease or occlusion of the major vessels. The inferior mesenteric artery was patent down to the thickened sigmoid.

After 10 days of intravenous hydrocortisone his symptoms were unchanged. Repeat flexible sigmoidoscopy was performed which showed mucosal inflammation and slough from the mid-rectum to sigmoid, again the lumen was noted to be stenosed, 'fixed' and firm on biopsy (figure 2).

In view of the lack of response to steroids and deterioration in the inflammatory indices (albumin $12 \mathrm{~g} / \mathrm{L}, \mathrm{CRP}$ $108 \mathrm{mg} / \mathrm{L}$ ) the patient was consented for a sigmoid colectomy. 


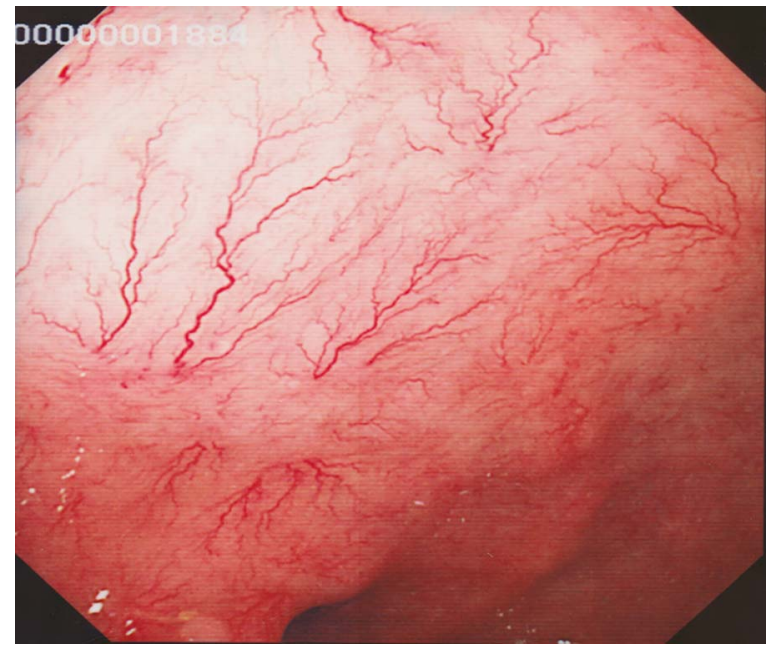

Figure 1 Enhanced vascular pattern and erythema in rectum.

At laparotomy the sigmoid colon (located on the right side of abdomen) was grossly thickened and ischaemic in appearance. The mesentery was also extremely thickened. A Hartmann's procedure was performed.

The resection specimen was grossly abnormal with venous congestion. Macroscopically there was a segment of infarction with congestion and ulceration. Microscopically there was evidence of myointimal hyperplasia by fibroplastic tissue resulting in diminution in the lumina of colonic and rectal veins and venules. Arteries showed evidence of modest atherosclerosis but no arteritis. There was no evidence of venous thrombosis of larger vessels (figures 3 and 4). A diagnosis of mesenteric inflammatory veno-occlusive disease (MIVOD) was made.

A screen for procoagulant conditions was negative. His clinical course was complicated by a postoperative collection which was drained via the rectal stump

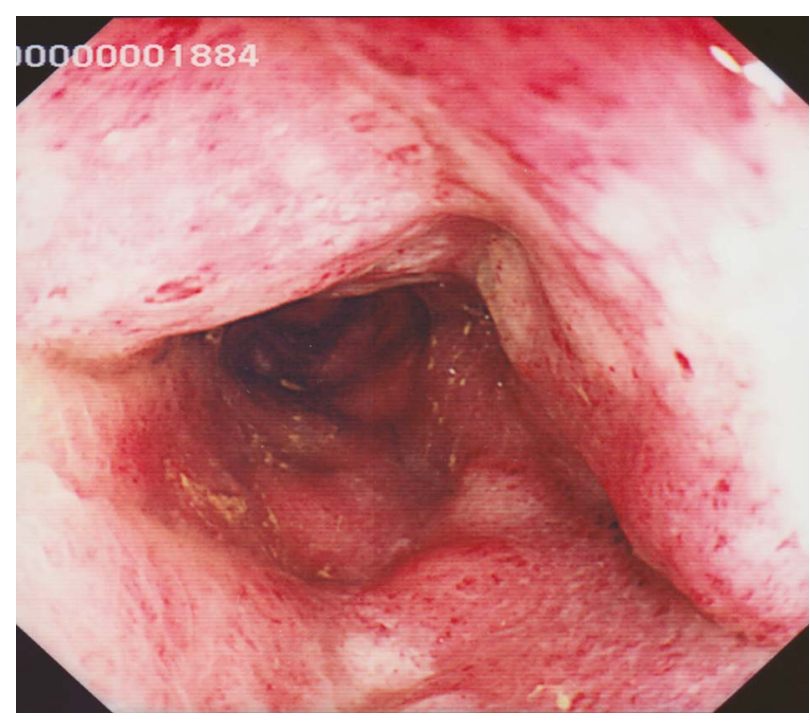

Figure 2 Narrowed 'rigid' sigmoid. under general anaesthesia. He was asymptomatic at 3 months follow-up and he underwent an elective reanastomosis at 6 months.

On reflection, the initial sequence of investigations could have been streamlined: barium enema is generally considered inferior to colonoscopy where tissue specimens can easily be obtained-although in this case mucosal biopsies were non-diagnostic. We also acknowledge the trial of intravenous steroids was prolonged, however even when there was profound biochemical deterioration the patient appeared clinically well without abdominal pain, peritonism or evidence of colonic dilatation.

\section{DISCUSSION}

Mesenteric ischaemia comprises a heterogeneous group of conditions. It is usually due to thromboembolic disease and associated with older age and typical vascular risk factors (see table 1).

MIVOD is an extremely rare venulitis affecting mesenteric vessels causing mesenteric and colonic ischaemia. There is debate as to whether it can be termed a true vasculitis. It was first reported in 1994. Flaherty et $a l^{1}$ described seven patients with intestinal ischaemia who all required surgical intervention. The resected specimens showed a characteristic phlebitis or venulitis affecting the veins and venules of the bowel and mesentery leading to ischaemic injury of the affected segment of bowel. In addition to the inflammatory infiltrate, three of seven patients also exhibited myointimal hyperplasia of the affected vessels. The authors proposed the term now used to describe this rare condition.

Other causes of mesenteric venous occlusion such as a hypercoagulable state, sepsis or trauma should be excluded if the diagnosis of MIVOD is being considered. The aetiology of MIVOD is unknown however

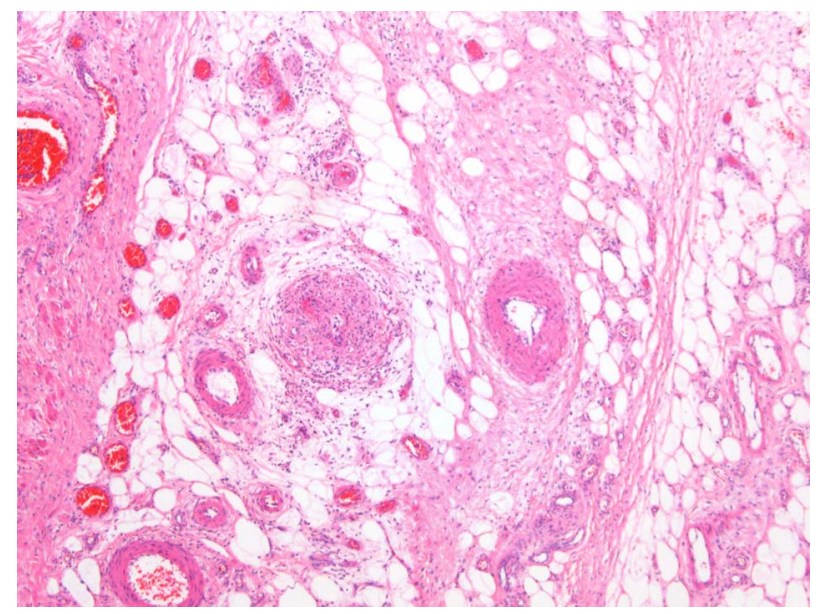

Figure 3 MIVOD—affected vein and normal artery. A subserosal vein (left), almost completely occluded and with a florid inflammatory cell infiltrate, contrasts with the adjacent artery which is normal. 


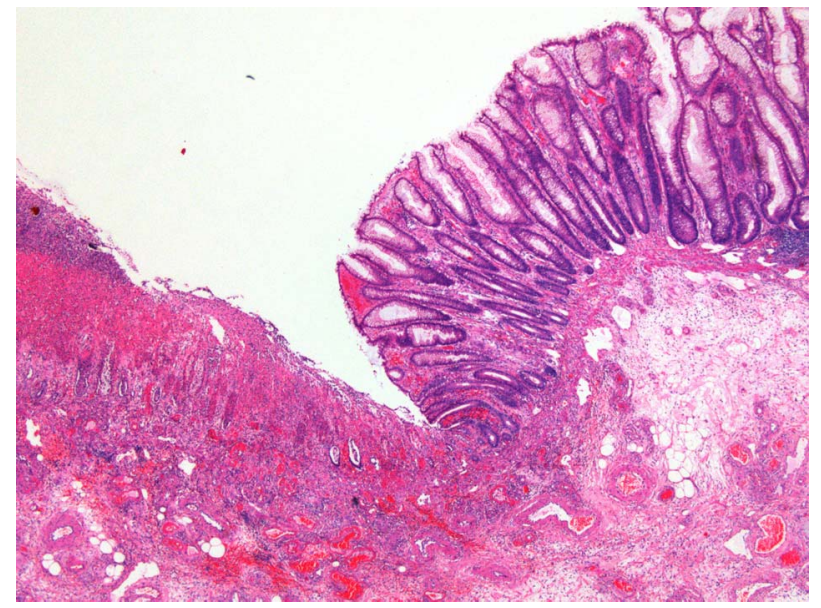

Figure 4 MIVOD typical venous ischaemia of colon. Extensive ulceration of the colon with typical features of venous ischaemia. There is a sharp cut-off, at the edge of the ulcer, to relatively normal colonic mucosa. Crypt bases of glands are discernible in the ulcerated area and these show 'withering' characteristic of ischaemia.

case reports implicate the antiphospholipid syndrome in one patient with subsequent $\mathrm{DVT}^{2}$; drugs including rutoside (an antioxidant), reserpine, methyldopa and amlodipine $^{3}$ and CMV infection. ${ }^{4}$

MIVOD tends to affect a localised segment of the colon but may also affect the small bowel. ${ }^{5}{ }^{6}$ Most reports describe a shorter duration of symptoms, typically mild in nature which may include nausea, anorexia, non-specific abdominal pain and constipation (diarrhoea is reported less commonly) ranging from days to weeks. ${ }^{7}$ This prodrome invariably culminates in presentation to emergency services with an acute abdomen. Fulminant colitis and the presence of skip lesions has also been reported which may contribute to initial confusion with Crohn's disease. ${ }^{8}$

\section{Table 1 Causes and characteristics of mesenteric ischaemia}

\begin{tabular}{|c|c|c|}
\hline Vessel & Diagnosis & Clinical context \\
\hline \multirow[t]{4}{*}{ Arterial } & $\begin{array}{l}\text { Superior mesenteric artery } \\
\text { embolism }\end{array}$ & Cardioembolism \\
\hline & $\begin{array}{l}\text { Superior mesenteric artery } \\
\text { thrombosis }\end{array}$ & $\begin{array}{l}\text { Atherosclerotic stenoses } \\
\text { Trauma } \\
\text { Infection }\end{array}$ \\
\hline & Non-occlusive ischaemia & $\begin{array}{l}\text { Atherosclerosis } \\
\text { Vasospasm } \\
\text { Shock }\end{array}$ \\
\hline & Vasculitis & $\begin{array}{l}\text { Systemic vasculitis (SLE, PAN, } \\
\text { Behcet's, Churg-Strauss) }\end{array}$ \\
\hline \multirow[t]{4}{*}{ Venous } & Mesenteric venous thrombosis & $\begin{array}{l}\text { Hypercoagulable states } \\
\text { Trauma } \\
\text { Pancreatitis } \\
\text { Portal hypertension }\end{array}$ \\
\hline & $\begin{array}{l}\text { Mesenteric inflammatory } \\
\text { veno-occlusive disease }\end{array}$ & Segmental colitis \\
\hline & $\begin{array}{l}\text { Idiopathic myointimal } \\
\text { hyperplasia of the mesenteric } \\
\text { veins }\end{array}$ & $\begin{array}{l}\text { Male adults, affects } \\
\text { rectosigmoid }\end{array}$ \\
\hline & $\begin{array}{l}\text { Idiopathic mesenteric } \\
\text { phlebosclerosis }\end{array}$ & $\begin{array}{l}\text { Chinese herbal medication is a } \\
\text { risk factor }\end{array}$ \\
\hline
\end{tabular}

PAN, polyarteritis nodosum; SLE, systemic lupus erythematosis.
In the early phase of MIVOD cross-sectional imaging and endoscopic examination tend to be noncontributory. Later, CT may show non-specific abnormalities such as bowel wall thickening, mesenteric fat stranding, and low volume lymphadenopathy. ${ }^{9}$

Colonic mucosal biopsies are too superficial to demonstrate the underlying disease process. Therefore the diagnosis can only be confirmed on histological examination of the resection specimen as in this case. Typical findings are venous congestion and infiltration.

Medical therapy is not effective. In the case described the inflammatory process was unaffected by steroid therapy. The efficacy of anticoagulation is unknown and we speculate it is unlikely to be effective when the primary pathology is an inflammatory infiltrate in the veins and venules. This is in contrast with large vessel thromboembolic disease affecting the mesenteric vessels where anticoagulation or thrombolysis may promote thrombus dissolution. MIVOD is treated surgically. Following resection the prognosis is extremely good; recurrence has only been reported once. ${ }^{6}$ It is unclear why the pathological process is localised and why recurrence is seldom observed.

Review of the literature reveals two other rare causes of non-thrombotic mesenteric venous stenosis or occlusion. Idiopathic myointimal hyperplasia of mesenteric veins follows a similar clinical course to MIVOD but appears to solely affect the left colon in adult men. ${ }^{10}$ Diagnosis is established following examination of the resection specimen which shows proliferation of smooth muscle causing focal thickening of the intima of mesenteric and intestinal mural veins. ${ }^{11}$ In contrast to MIVOD, an inflammatory infiltrate is not present.

Idiopathic mesenteric phlebosclerosis causes a similar, gradual onset of chronic symptoms such as described in this case of MIVOD. Idiopathic mesenteric phlebosclerosis is characterised by calcifications in the small mesenteric veins and intramural branches. It affects the right colon which has darkened mucosa and ulceration at colonoscopy. In a case series from Japan patients were treated with subtotal colectomy. Microscopic examination of the resection specimens showed marked fibrous thickening of the venous walls with calcification, marked submucosal fibrosis, deposition of collagen in the mucosa, and foamy macrophages within the vessel walls. Most of the reported cases are from Asia and long term herbal medications have been suggested as a cause. ${ }^{12}$

In contrast to vasculitis involving the gastrointestinal tract, MIVOD, idiopathic myointimal hyperplasia of mesenteric veins and phlebosclerosis are more difficult to diagnose as these conditions do not present with clues that a multisystem vasculitic process is present and a histological diagnosis can only be made following surgery. Hence there is often a delay in diagnosis. 


\section{CONCLUSION}

MIVOD is a rare cause of intestinal ischaemia occurring in individuals without prior GI disease and is not associated with risk factors for venous or arterial disease. It should be considered when other causes of mesenteric ischaemia have been excluded and in cases of colitis not responding to standard medical therapy. It may mimic IBD however colonic mucosal biopsies demonstrate oedema only. The diagnosis is established on examination of the resection specimen. Following surgical resection the prognosis is extremely good.

This case highlights the utility of repeating investigations when the diagnosis is unclear and in this situation sequential flexible sigmoidoscopy was useful with respect to decisions regarding surgery.

Contributors LROA is the first author of the paper. Coauthors are MS, NS and JB who reviewed and amended the paper.

Competing interests None.

Patient consent Obtained.

Provenance and peer review Not commissioned; externally peer reviewed.

\section{REFERENCES}

1 Flaherty MJ, Lie JT, Haggitt RC. Mesenteric inflammatory veno-occlusive disease. A seldom recognized cause of intestinal ischaemia. Am J Surg Pathol 1994;18:779-84.

2 Gül A, Inanç M, Ocal L, et al. Primary antiphospholipid syndrome associated with mesenteric inflammatory veno-occlusive disease. Clin Rheumatol 1996;15:207-10.
3 Saraga EP, Costa J. Idiopathic entero-colic lymphocytic phlebitis. A cause of ischemic intestinal necrosis. Am J Surg Pathol 1989;13:303-8.

4 Ailani RK, Simms R, Caracioni AA, et al. Extensive mesenteric inflammatory veno-occlusive disease of unknown etiology after primary cytomegalovirus infection: first case. Am J Gastroenterol 1997;92:1216-18.

5 Eryigit E, Hoentjen F, Barbe E, et al. Intestinal ischaemia caused by mesenteric inflammatory veno-occlusive disease. Neth J Med 2008;66:486-8.

6 Tempia-Caliera AA, Renzulli P, Z'graggen K, et al. Mesenteric inflammatory veno-occlusive disease: a rare cause of intestinal ischaemia. The first description of recurrent disease. Digestion 2002;66:262-4.

$7 \mathrm{Hu}$ JC, Forshaw MJ, Thebe P, et al. Mesenteric inflammatory veno-occlusive disease as a cause of acute abdomen: report of five cases. Surg Today 2005;35:961-4.

8 Canavan JB, Coss A, Leader M, et al. Acute fulminant colitis caused by idiopathic mesenteric inflammatory veno-occlusive disease. Case Rep Gastroenterol 2007;1:152-6.

9 Verwimp W, Feyaerts F, Vanbeckevoort D. Mesenteric inflammatory veno-occlusive disease (MIVOD). Images in clinical radiology. JBR-BTR 2007;90:546.

10 Kao PC, Vecchio JA, Hyman NH, et al. Idiopathic myointimal hyperplasia of mesenteric veins: a rare mimic of idiopathic inflammatory bowel disease. J Clin Gastroenterol 2005;39:704-8.

11 Genta RM, Haggitt RC. Idiopathic myointimal hyperplasia of mesenteric veins. Gastroenterology 1991;101:533-9.

12 Iwashita A, Yao T, Schlemper RJ, et al. Mesenteric phlebosclerosis: a new disease entity causing ischemic colitis. Dis Colon Rectum 2003;46:209-20. 